\title{
A Gentle (without Chopping) Approach to the Full Kostant-Toda Lattice
}

Pantelis A. DAMIANOU ${ }^{\dagger}$ and Franco MAGRI ${ }^{\ddagger}$

$\dagger$ Department of Mathematics and Statistics, University of Cyprus, 1678, Nicosia, Cyprus E-mail: damianou@ucy.ac.cy

URL: http://www.ucy.ac.cy/ damianou/

$\ddagger$ Department of Mathematics, University of Milano Bicocca, Via Corsi 58, I 20126 Milano, Italy

E-mail: magri@matapp.unimib.it

Received September 22, 2005, in final form October 24, 2005; Published online October 25, 2005

Original article is available at http://www.emis.de/journals/SIGMA/2005/Paper010/

Abstract. In this paper we propose a new algorithm for obtaining the rational integrals of the full Kostant-Toda lattice. This new approach is based on a reduction of a bi-Hamiltonian system on $g l(n, \mathbb{R})$. This system was obtained by reducing the space of maps from $Z_{n}$ to $G L(n, \mathbb{R})$ endowed with a structure of a pair of Lie-algebroids.

Key words: full Kostant-Toda lattice; integrability; bi-Hamiltonian structure

2000 Mathematics Subject Classification: 37J35; 70H06

\section{Introduction}

The Toda lattice is arguably the most fundamental and basic of all finite dimensional integrable systems. It has various intriguing connections with other parts of mathematics and physics.

The Hamiltonian of the Toda lattice is given by

$$
H\left(q_{1}, \ldots, q_{N}, p_{1}, \ldots, p_{N}\right)=\sum_{i=1}^{N} \frac{1}{2} p_{i}^{2}+\sum_{i=1}^{N-1} e^{q_{i}-q_{i+1}} .
$$

Equation (1) is known as the classical, finite, non-periodic Toda lattice to distinguish the system from the many and various other versions, e.g., the relativistic, quantum, periodic etc. This system was investigated in [9, 10, 15, 19, 22, 23, 25] and numerous of other papers; see [4] for a more extensive bibliography.

Hamilton's equations become

$$
\begin{aligned}
& \dot{q}_{j}=p_{j}, \\
& \dot{p}_{j}=e^{q_{j-1}-q_{j}}-e^{q_{j}-q_{j+1}} .
\end{aligned}
$$

The system is integrable. One can find a set of independent functions $\left\{H_{1}, \ldots, H_{N}\right\}$ which are constants of motion for Hamilton's equations. To determine the constants of motion, one uses Flaschka's transformation:

$$
a_{i}=\frac{1}{2} e^{\frac{1}{2}\left(q_{i}-q_{i+1}\right)}, \quad b_{i}=-\frac{1}{2} p_{i} .
$$

Then

$$
\dot{a}_{i}=a_{i}\left(b_{i+1}-b_{i}\right), \quad \dot{b}_{i}=2\left(a_{i}^{2}-a_{i-1}^{2}\right) .
$$


These equations can be written as a Lax pair $\dot{L}=[B, L]$, where $L$ is the Jacobi matrix

$$
L=\left(\begin{array}{cccccc}
b_{1} & a_{1} & 0 & \cdots & \cdots & 0 \\
a_{1} & b_{2} & a_{2} & \cdots & & \vdots \\
0 & a_{2} & b_{3} & \ddots & & \\
\vdots & & \ddots & \ddots & & \vdots \\
\vdots & & & \ddots & \ddots & a_{N-1} \\
0 & \cdots & & \cdots & a_{N-1} & b_{N}
\end{array}\right),
$$

and $B$ is the skew-symmetric part of $L$. This is an example of an isospectral deformation; the entries of $L$ vary over time but the eigenvalues remain constant. It follows that the functions $H_{i}=\frac{1}{i} \operatorname{tr} L^{i}$ are constants of motion.

Note that the Lax pair (4) has the form

$$
\dot{L}(t)=[P L(t), L(t)]
$$

where $P$ denotes the projection onto the skew-symmetric part in the decomposition of $L$ into skew-symmetric plus lower triangular.

The Toda lattice was generalized in several directions.

We mention the Bogoyavlensky-Toda lattices which generalize the Toda lattice (which corresponds to a root system of type $A_{n}$ ) to other simple Lie groups. This generalization is due to Bogoyavlensky [1]. These systems were studied extensively in [16] where the solution of the systems was connected intimately with the representation theory of simple Lie groups.

Another generalization is due to Deift, Li, Nanda and Tomei [5] who showed that the system remains integrable when $L$ is replaced by a full (generic) symmetric $n \times n$ matrix.

Another variation is the full Kostant-Toda lattice (FKT) $[6,11,24]$. We briefly describe the system: In [16] Kostant conjugates the matrix $L$ in (4) by a diagonal matrix to obtain a matrix of the form

$$
L=\left(\begin{array}{cccccc}
b_{1} & 1 & 0 & \cdots & \cdots & 0 \\
a_{1} & b_{2} & 1 & \ddots & & \vdots \\
0 & a_{2} & b_{3} & \ddots & & \vdots \\
\vdots & \ddots & \ddots & \ddots & & 0 \\
\vdots & & & \ddots & \ddots & 1 \\
0 & \cdots & \cdots & 0 & a_{n-1} & b_{n}
\end{array}\right) .
$$

The equations take the form

$$
\dot{X}(t)=[X(t), P X(t)]
$$

where $P$ is the projection onto the strictly lower triangular part of $X(t)$. This form is convenient in applying Lie theoretic techniques to describe the system.

To obtain the full Kostant-Toda lattice we fill the lower triangular part of $L$ in (4) with additional variables. ( $P$ is again the projection onto the strictly lower part of $X(t)$ ). So, using the notation from $[6,11]$ and [24]

$$
\dot{X}(t)=[X(t), P X(t)],
$$


where $X$ is in $\epsilon+B_{-}$and $P X$ is in $N_{-} . B_{-}$is the Lie algebra of lower triangular matrices and $N_{-}$is the Lie algebra of strictly lower triangular matrices. The fixed matrix $\epsilon$ has a general form in terms of root systems:

$$
\epsilon=\sum_{\alpha \in \Delta} x_{\alpha}
$$

where $\Delta$ denotes the set of simple roots. In fact the FKT lattice itself can easily be generalized for each simple Lie group; see $[2,6]$.

In the case of $\operatorname{sl}(4, \mathbb{C})$ the matrix $X$ has the form

$$
X=\left(\begin{array}{cccc}
f_{1} & 1 & 0 & 0 \\
g_{1} & f_{2} & 1 & 0 \\
h_{1} & g_{2} & f_{3} & 1 \\
k_{1} & h_{2} & g_{3} & f_{4}
\end{array}\right),
$$

with $\sum_{i} f_{i}=0$.

The functions $H_{i}=\frac{1}{i} \operatorname{Tr} X^{i}$ are still in involution but they are not enough to ensure integrability. This is a crucial point: the existence of a Lax pair does not guarantee integrability. There are, however, additional integrals which are rational functions of the entries of $X$. The method used to obtain these additional integrals is called chopping and was used originally in [5] for the full symmetric Toda and later in [6] for the case of the full Kostant-Toda lattice.

In this paper we use a different method of obtaining these rational integrals which does not involve chopping. This method uses a reduction of a bi-Hamiltonian system on $g l(N, \mathbb{R})$, a system which was first defined in [20, 21]. In [21] A. Meucci presents the bi-Hamiltonian structure of $\mathrm{Toda}_{3}$, a dynamical system studied by Kupershmidt in [17] as a reduction of the KP hierarchy. Meucci derives this structure by a suitable restriction of the set of maps from $Z_{d}$, where $Z_{d}$ is the cyclic group of order $d$, to $G L(3, \mathbb{R})$, in the context of Lie algebroids.

In [20] the bi-Hamiltonian structure of the periodic Toda lattice is investigated by the reduction process described above using maps from $Z_{d}$ to $G L(2, \mathbb{R})$. This approach parallels the work of [7] where the continuous analog of the Toda lattice is studied, namely the KdV. If instead the target space is $g l(3, \mathbb{R})$ one obtains the Boussineq hierarchy. The work of Meucci is a discrete version of this approach. If one generalizes the cases $N=2,3$, i.e. consider maps from $Z_{d}$ to $G L(N, \mathbb{R})$ the resulting system will be denoted by $\operatorname{Toda}_{N}$. In the present paper we use the results of $[20,21]$ as a starting point. We begin with the bi-Hamiltonian system obtained on $g l(N, \mathbb{R})$ in the particular case $d=N$ and use a further reduction to obtain the dynamics of the full Kostant-Toda lattice. We then propose a new algorithm which produces all the rational integrals for the FKT lattice without using chopping. We present this algorithm by specific examples $(N=3,4)$ in Section 4 . Sections 2 and 3 contain a general review of the old methods and results on integrability and bi-Hamiltonian structure of the FKT lattice.

\section{Integrability of the FKT lattice}

Let $\mathcal{G}=\operatorname{sl}(n)$, the Lie algebra of $n \times n$ matrices of trace zero. Using the decomposition $\mathcal{G}=B_{+} \oplus N_{-}$we can identify $B_{+}^{*}$ with the annihilator of $N_{-}$with respect to the Killing form. This annihilator is $B_{-}$. Thus we can identify $B_{+}^{*}$ with $B_{-}$and therefore with $\epsilon+B_{-}$as well.

The Lie-Poisson bracket in the case of $s l(4, \mathbb{C})$ is given by the following defining relations:

$$
\begin{aligned}
& \left\{g_{i}, g_{i+1}\right\}=h_{i}, \\
& \left\{g_{i}, f_{i}\right\}=-g_{i},
\end{aligned}
$$




$$
\begin{aligned}
& \left\{g_{i}, f_{i+1}\right\}=g_{i}, \\
& \left\{h_{i}, f_{i}\right\}=-h_{i}, \\
& \left\{h_{i}, f_{i+2}\right\}=h_{i}, \\
& \left\{g_{1}, h_{2}\right\}=k_{1}, \\
& \left\{g_{3}, h_{1}\right\}=-k_{1}, \\
& \left\{k_{1}, f_{1}\right\}=-k_{1}, \\
& \left\{k_{1}, f_{4}\right\}=k_{1} .
\end{aligned}
$$

All other brackets are zero. Actually, we calculated the brackets on $g l(4, \mathbb{C})$; the trace of $X$ now becomes a Casimir. The Hamiltonian in this bracket is $H_{2}=\frac{1}{2} \operatorname{Tr} X^{2}$.

Remark 1. If we use a more conventional notation for the matrix $X$, i.e. $x_{i j}$ for $i \geq j, x_{i i+1}=1$, and all other entries zero, then the bracket is simply

$$
\left\{x_{i j}, x_{k l}\right\}=\delta_{l i} x_{k j}-\delta_{j k} x_{i l} .
$$

The functions $H_{i}=\frac{1}{i} \operatorname{Tr} X^{i}$ are still in involution but they are not enough to ensure integrability. There are, however, additional integrals and the interesting feature of this system is that the additional integrals turn out to be rational functions of the entries of $X$. We describe the constants of motion following references [6, 11, 24].

For $k=0, \ldots,\left[\frac{(n-1)}{2}\right]$, denote by $(X-\lambda \mathrm{Id})_{(k)}$ the result of removing the first $k$ rows and last $k$ columns from $X-\lambda \mathrm{Id}$, and let

$$
\operatorname{det}(X-\lambda \mathrm{Id})_{(k)}=E_{0 k} \lambda^{n-2 k}+\cdots+E_{n-2 k, k} .
$$

Set

$$
\frac{\operatorname{det}(X-\lambda \mathrm{Id})_{(k)}}{E_{0 k}}=\lambda^{n-2 k}+I_{1 k} \lambda^{n-2 k-1}+\cdots+I_{n-2 k, k} .
$$

The functions $I_{r k}, r=1, \ldots, n-2 k$, are constants of motion for the FKT lattice.

Example 1. We consider in detail the $g l(3, \mathbb{C})$ case:

$$
X=\left(\begin{array}{lll}
f_{1} & 1 & 0 \\
g_{1} & f_{2} & 1 \\
h_{1} & g_{2} & f_{3}
\end{array}\right)
$$

Taking $H_{2}=\frac{1}{2} \operatorname{tr} X^{2}$ as the Hamiltonian, and the above Poisson bracket

$$
\dot{x}=\left\{H_{2}, x\right\}
$$

gives the following equations:

$$
\begin{aligned}
& \dot{f}_{1}=-g_{1}, \\
& \dot{f}_{2}=g_{1}-g_{2}, \\
& \dot{f}_{3}=g_{2}, \\
& \dot{g}_{1}=g_{1}\left(f_{1}-f_{2}\right)-h_{1}, \\
& \dot{g}_{2}=g_{2}\left(f_{2}-f_{3}\right)+h_{1}, \\
& \dot{h}_{1}=h_{1}\left(f_{1}-f_{3}\right)
\end{aligned}
$$


Note that $H_{1}=f_{1}+f_{2}+f_{3}$ while $H_{2}=\frac{1}{2}\left(f_{1}^{2}+f_{2}^{2}+f_{3}^{2}\right)+g_{1}+g_{2}$. These equations can be written in Lax pair form, $\dot{X}=[B, X]$, by taking

$$
B=\left(\begin{array}{ccc}
0 & 0 & 0 \\
g_{1} & 0 & 0 \\
h_{1} & g_{2} & 0
\end{array}\right)
$$

The chopped matrix is given by

$$
\left(\begin{array}{cc}
g_{1} & f_{2}-\lambda \\
h_{1} & g_{2}
\end{array}\right)
$$

The determinant of this matrix is $h_{1} \lambda+g_{1} g_{2}-h_{1} f_{2}$ and we obtain the rational integral

$$
I_{11}=\frac{g_{1} g_{2}-h_{1} f_{2}}{h_{1}} .
$$

Note that the phase space is six dimensional, we have two Casimirs $\left(H_{1}, I_{11}\right)$ and the functions $\left(H_{2}, H_{3}\right)$ are enough to ensure integrability.

Example 2. In the case of $g l(4, \mathbb{C})$ the additional integral is

$$
I_{21}=\frac{g_{1} g_{2} g_{3}-g_{1} f_{3} h_{2}-f_{2} g_{3} h_{1}+h_{1} h_{2}}{k_{1}}+f_{2} f_{3}-g_{2} \text {. }
$$

and

$$
I_{11}=\frac{g_{1} h_{2}+g_{3} h_{1}}{k_{1}}-f_{2}-f_{3}
$$

is a Casimir.

In this example the phase space is ten dimensional, we have two Casimirs $\left(H_{1}, I_{11}\right)$ and the functions $\left(H_{2}, H_{3}, H_{4}, I_{21}\right)$ are independent and pairwise in involution.

\section{Bi-Hamiltonian structure}

We recall the definition and basic properties of master symmetries following Fuchssteiner [13]. Consider a differential equation on a manifold $M$ defined by a vector field $\chi$. We are mostly interested in the case where $\chi$ is a Hamiltonian vector field. A vector field $Z$ is a symmetry of the equation if

$$
[Z, \chi]=0 .
$$

A vector field $Z$ is called a master symmetry if

$$
[[Z, \chi], \chi]=0
$$

but

$$
[Z, \chi] \neq 0
$$

Master symmetries were first introduced by Fokas and Fuchssteiner in [12] in connection with the Benjamin-Ono Equation.

A bi-Hamiltonian system is defined by specifying two Hamiltonian functions $H_{1}, H_{2}$ and two Poisson tensors $\pi_{1}$ and $\pi_{2}$, that give rise to the same Hamiltonian equations. Namely, $\pi_{1} \nabla H_{2}=\pi_{2} \nabla H_{1}$. The notion of bi-Hamiltonian system was introduced in [18] in 1978. 
Another idea that will be useful is the Gelfand-Zakharevich scheme for a pencil of Poisson tensors. Consider a bi-Hamiltonian system given by two compatible Poisson tensors $\pi_{1}, \pi_{2}$. Compatible means that the pencil

$$
\{,\}_{\lambda}=\{,\}_{\pi_{1}}+\lambda\{,\}_{\pi_{2}}
$$

is Poisson for each value of the real parameter $\lambda$. Gelfand and Zakharevich in [14] consider the special case in which the pencil possesses only one Casimir. Under some mild conditions they prove the following:

Let

$$
F_{\lambda}=F_{0}+\lambda F_{1}+\cdots+\lambda^{n} F_{n} .
$$

Then $F_{0}$ is a Casimir for $\pi_{1}, F_{n}$ is a Casimir for $\pi_{2}$ and, in addition, the functions $F_{0}, F_{1}, \ldots, F_{n}$ are in involution with respect to both brackets $\pi_{1}$ and $\pi_{2}$.

We now return to the FKT lattice. We want to define a second bracket $\pi_{2}$ so that $H_{1}$ is the Hamiltonian and

$$
\pi_{2} \nabla H_{1}=\pi_{1} \nabla H_{2} \text {. }
$$

i.e. we want to construct a bi-Hamiltonian pair. We will achieve this by finding a master symmetry $X_{1}$ so that

$$
X_{1}\left(\operatorname{Tr} X^{i}\right)=i \operatorname{Tr} X^{i+1} .
$$

We construct $X_{1}$ by considering the equation

$$
\dot{X}=[Y, X]+X^{2} \text {. }
$$

We choose $Y$ in such a way that the equation is consistent. One solution is

$$
Y=\sum_{i=1}^{n} \alpha_{i} E_{i i}+\sum_{i=1}^{n-1} \beta_{i} E_{i, i+1},
$$

where $\beta_{i}=i, \alpha_{i}=i f_{i}+\sum_{k=1}^{i-1} f_{k}$.

The vector field $X_{1}$ is defined by the right hand side of (9).

For example, in $g l(4, \mathbb{C})$ the components of $X_{1}$ are:

$$
\begin{aligned}
& X_{1}\left(f_{1}\right)=2 g_{1}+f_{1}^{2}, \\
& X_{1}\left(f_{2}\right)=3 g_{2}+f_{2}^{2}, \\
& X_{1}\left(f_{3}\right)=-g_{2}+4 g_{3}+f_{3}^{2}, \\
& X_{1}\left(f_{4}\right)=-2 g_{3}+f_{4}^{2}, \\
& X_{1}\left(g_{1}\right)=3 h_{1}+g_{1} f_{1}+3 g_{1} f_{2}, \\
& X_{1}\left(g_{2}\right)=4 h_{2}+4 g_{2} f_{3}, \\
& X_{1}\left(g_{3}\right)=-h_{2}-g_{3} f_{3}+5 g_{3} f_{4}, \\
& X_{1}\left(h_{1}\right)=g_{1} g_{2}+4 k_{1}+h_{1} f_{1}+h_{1} f_{2}+4 h_{1} f_{3}, \\
& X_{1}\left(h_{2}\right)=g_{2} g_{3}+h_{2} f_{3}+5 h_{2} f_{4}, \\
& X_{1}\left(k_{1}\right)=g_{3} h_{1}+g_{1} h_{2}+k_{1} f_{1}+k_{1} f_{2}+k_{1} f_{3}+5 k_{1} f_{4} .
\end{aligned}
$$


The second bracket $\pi_{2}$ is defined by taking the Lie derivative of $\pi_{1}$ in the direction of $X_{1}$. The bracket $\pi_{2}$ is at most quadratic, i.e. in the case $n=4$

$$
\begin{aligned}
& \left\{g_{i}, g_{i+1}\right\}=g_{i} g_{i+1}+h_{i} f_{i+1}, \\
& \left\{g_{i}, h_{i}\right\}=g_{i} h_{i}, \\
& \left\{g_{i+1}, h_{i}\right\}=-g_{i+1} h_{i}, \\
& \left\{g_{i}, f_{i}\right\}=-g_{i} f_{i}, \\
& \left\{g_{i}, f_{i+1}\right\}=g_{i} f_{i+1}, \\
& \left\{g_{i}, f_{i+2}\right\}=h_{i}, \\
& \left\{g_{i+1}, f_{i}\right\}=-h_{i}, \\
& \left\{h_{i}, f_{i}\right\}=-h_{i} f_{i}, \\
& \left\{h_{i}, f_{i+2}\right\}=h_{i} f_{i+2}, \\
& \left\{f_{i}, f_{i+1}\right\}=g_{i} .
\end{aligned}
$$

This bracket was first obtained in [3] using the method described above (i.e. master symmetries). A closed expression for this bracket was obtained later by Faybusovich and Gekhman in [8]. It was obtained using $R$-matrices and the expression takes the following simple form:

$$
\left\{x_{i j}, x_{k l}\right\}= \begin{cases}\operatorname{sign}(k-i) x_{i j} x_{k j} & \text { if } j=l, \\ x_{i j} x_{i l} & \text { if } k=i, \\ x_{i j} x_{k l}+x_{i l} x_{k j} & \text { if } i<k \leq j, \\ x_{i l} & \text { if } k=j+1 .\end{cases}
$$

As we will see in the next section, there is a linear and a quadratic bracket on $g l(n, \mathbb{R})$ whose restriction to the FKT lattice coincides with the brackets $\pi_{1}, \pi_{2}$.

\section{A new approach}

We already mentioned in the introduction that our starting point is the work of A. Meucci [20, 21]. His work is a discrete analogue of a procedure that produces the KdV, Boussineq and Gelfand-Dickey hierarcies [7]. For example, the KdV is bi-Hamiltonian and it can be obtained by reducing the space of $C^{\infty}$ maps from $S^{1}$ to $g l(2, \mathbb{R})$. If one considers the space of maps from $S^{1}$ to $g l(3, \mathbb{R})$ the Boussineq hierarchy is obtained. In $[20,21]$ the discrete version of the procedure is considered. The circle, $S^{1}$, is replaced by the cyclic group $\mathbb{Z}_{d}$ and one considers maps from $\mathbb{Z}_{d}$ to $G L(N, \mathbb{R})$. One obtains, after reduction, equations that are bi-Hamiltonian. In the case $N=2$ the resulting system is the periodic Toda lattice and for $N=3$ a system studied by Kupershmidt in [17]. We will not get into the details of the procedure but rather we will use the results as our starting point for our own purposes. We will content with a short outline of the constructions of [20] and [21]. The basic object is the space of maps from $\mathbb{Z}_{d}$ to $G L(N, \mathbb{R})$. This space is endowed with a structure consisting of a Poisson manifold together with a pair of Lie-algebroids suitably related. The next step is a Marsden-Ratiu type of reduction and the result is a bi-Hamiltonian system with a pair of Poisson structures on $g l(N, \mathbb{R})$. The Lax pair of the resulting system contains two spectral parameters and the theory of Gelfand-Zakarevich applies. The system turns out to be integrable with the required number of integrals. We give explicit formulas that we have computed from [20] in the case $N=3$ both for the pair of Poisson brackets, the Lax pair and the integrals of motion. In the case $N=4$ we display the Lax pair and the polynomial integrals of motion. To obtain the FKT lattice one has to perform a further reduction to the phase space of the FKT lattice and to obtain the rational integrals we propose a new algorithm which is the central new result of our paper. At the present we do not have a Lie algebraic interpretation of this algorithm. We illustrate with two examples: 
Example 3 (The $g l(3, \mathbb{C})$ case). The phase space of the system obtained by the procedure of Meucci is nine dimensional, i.e. matrices of the form

$$
\left(\begin{array}{lll}
f_{1} & h_{2} & g_{3} \\
g_{1} & f_{2} & h_{3} \\
h_{1} & g_{2} & f_{3}
\end{array}\right)
$$

We compute the pair of Poisson brackets on the extended space with variables

$$
\left\{f_{1}, f_{2}, f_{3}, g_{1}, g_{2}, g_{3}, h_{1}, h_{2}, h_{3}\right\} .
$$

The Lie-Poisson bracket is defined by the following structure matrix

$$
\left(\begin{array}{ccccccccc}
0 & 0 & 0 & -g_{1} & 0 & g_{3} & -h_{1} & h_{2} & 0 \\
0 & 0 & 0 & g_{1} & -g_{2} & 0 & 0 & -h_{2} & h_{3} \\
0 & 0 & 0 & 0 & g_{2} & -g_{3} & h_{1} & 0 & -h_{3} \\
g_{1} & -g_{1} & 0 & 0 & -h_{1} & h_{3} & 0 & 0 & 0 \\
0 & g_{2} & -g_{2} & h_{1} & 0 & -h_{2} & 0 & 0 & 0 \\
-g_{3} & 0 & g_{3} & -h_{3} & h_{2} & 0 & 0 & 0 & 0 \\
h_{1} & 0 & -h_{1} & 0 & 0 & 0 & 0 & 0 & 0 \\
-h_{2} & h_{2} & 0 & 0 & 0 & 0 & 0 & 0 & 0 \\
0 & -h_{3} & h_{3} & 0 & 0 & 0 & 0 & 0 & 0
\end{array}\right)
$$

and the quadratic Poisson bracket is defined by $A-A^{t}$ where $A$ is the matrix

$$
\left(\begin{array}{ccccccccc}
0 & g_{1} & -g_{3} & -g_{1} f_{1} & h_{1}-h_{2} & g_{3} f_{1} & -h_{1} f_{1} & h_{2} f_{1} & 0 \\
0 & 0 & g_{2} & g_{1} f_{2} & -g_{2} f_{2} & h_{2}-h_{3} & 0 & -h_{2} f_{2} & h_{3} f_{2} \\
0 & 0 & 0 & h_{3}-h_{1} & g_{2} f_{3} & -g_{3} f_{3} & h_{1} f_{3} & 0 & -h_{3} f_{3} \\
0 & 0 & 0 & 0 & -h_{1} f_{2}-g_{1} g_{2} & h_{3} f_{1}+g_{1} g_{3} & -g_{1} h_{1} & 0 & g_{1} h_{3} \\
0 & 0 & 0 & 0 & 0 & -h_{2} f_{3}-g_{2} g_{3} & g_{2} h_{1} & -g_{2} h_{2} & 0 \\
0 & 0 & 0 & 0 & 0 & 0 & 0 & g_{3} h_{2} & -g_{3} h_{3} \\
0 & 0 & 0 & 0 & 0 & 0 & 0 & 0 & 0 \\
0 & 0 & 0 & 0 & 0 & 0 & 0 & 0 & 0 \\
0 & 0 & 0 & 0 & 0 & 0 & 0 & 0 & 0
\end{array}\right) .
$$

The Lax matrix with two spectral parameters is given by

$$
L_{\lambda, \mu}=\left(\begin{array}{ccc}
\left(f_{1}+\lambda\right) \mu^{2} & h_{2}-\mu^{3} & \mu g_{3} \\
\mu g_{1} & \left(f_{2}+\lambda\right) \mu^{2} & h_{3}-\mu^{3} \\
h_{1}-\mu^{3} & \mu g_{2} & \left(f_{3}+\lambda\right) \mu^{2}
\end{array}\right) .
$$

Let $p(\lambda, \mu)=\operatorname{det} L_{\mu, \lambda}$. Write

$$
p(\lambda, \mu)=-\mu^{9}+c_{2}(\lambda) \mu^{6}+c_{1}(\lambda) \mu^{3}+c_{0}(\lambda) .
$$

Then

$$
c_{2}(\lambda)=\lambda^{3}+k_{2} \lambda^{2}+k_{1} \lambda+k_{0},
$$

where

$$
\begin{aligned}
& k_{2}=f_{1}+f_{2}+f_{3}, \\
& k_{1}=f_{1} f_{2}+f_{1} f_{3}+f_{2} f_{3}+g_{1}+g_{2}+g_{3}, \\
& k_{0}=f_{1} f_{2} f_{3}+f_{1} g_{2}+f_{2} g_{3}+f_{3} g_{1}+h_{1}+h_{2}+h_{3}
\end{aligned}
$$


and

$$
c_{1}(\lambda)=l_{1} \lambda+l_{0}
$$

where

$$
\begin{aligned}
& l_{1}=-h_{3} g_{2}-g_{3} h_{1}-h_{2} g_{1}, \\
& l_{0}=-f_{1} g_{2} h_{3}+g_{1} g_{3} g_{2}-g_{1} h_{2} f_{3}-h_{1} g_{3} f_{2}-h_{2} h_{3}-h_{3} h_{1}-h_{1} h_{2} .
\end{aligned}
$$

Finally,

$$
c_{0}(\lambda)=h_{1} h_{2} h_{3}
$$

The functions $k_{i}, l_{i}, c_{0}$ are all in involution in the Lie-Poisson bracket. The functions $k_{2}, l_{1}$, $c_{0}$ are all Casimirs.

In the quadratic bracket $k_{i}, l_{i}$, and $c_{0}$ are all in involution. $k_{0}, l_{0}$ and $c_{0}$ are Casimirs.

Let

$$
l=\frac{l_{0}}{l_{1}}=\frac{-f_{1} g_{2} h_{3}+g_{1} g_{3} g_{2}-g_{1} h_{2} f_{3}-h_{1} g_{3} f_{2}-h_{2} h_{3}-h_{3} h_{1}-h_{1} h_{2}}{-h_{3} g_{2}-g_{3} h_{1}-h_{2} g_{1}} .
$$

Setting $h_{2}=h_{3}=0$ in $l$ we obtain $I_{11}(8)$.

Example 4 (The $g l(4, \mathbb{C})$ case). In order to give a complete comparison of the previous and the present method of obtaining the rational invariants we consider first integrability using chopping.

We consider the matrix $L$ given by

$$
L=\left(\begin{array}{cccc}
f_{1} & -1 & 0 & 0 \\
g_{1} & f_{2} & -1 & 0 \\
h_{1} & g_{2} & f_{3} & -1 \\
k_{1} & h_{2} & g_{3} & f_{4}
\end{array}\right)
$$

Note that we are using $-\epsilon$ instead of $\epsilon$ in order to get the integrals to match exactly. In this case the chopped matrix has the form

$$
C h_{1}(\lambda)=\left(\begin{array}{ccc}
g_{1} & f_{2}-\lambda & -1 \\
h_{1} & g_{2} & f_{3}-\lambda \\
k_{1} & h_{2} & g_{3}
\end{array}\right) .
$$

The characteristic polynomial has the form

$$
k_{1} \lambda^{2}+\left(g_{1} h_{2}+h_{1} g_{3}-k_{1} f_{2}-k_{1} f_{3}\right) \lambda+g_{1} g_{3} g_{2}-g_{1} h_{2} f_{3}-h_{1} f_{2} g_{3}-h_{1} h_{2}+k_{1} f_{2} f_{3}+k_{1} g_{2} \text {. }
$$

We obtain the following two rational invariants

$$
i_{11}=\frac{h_{2} g_{1}+g_{3} h_{1}}{k 1}-\left(f_{2}+f_{3}\right)
$$

and

$$
i_{21}=\frac{g_{1} g_{3} g_{2}-g_{1} h_{2} f_{3}-h_{1} f_{2} g_{3}-h_{1} h_{2}}{k_{1}}+f_{2} f_{3}+g_{2}
$$


We now turn to the gentle approach, i.e. integrability without chopping. Consider the following Lax pair with two spectral parameters:

$$
L_{\lambda, \mu}=\left(\begin{array}{cccc}
\left(f_{1}+\lambda\right) \mu^{3} & k_{2}-\mu^{4} & h_{3} \mu & g_{4} \mu^{2} \\
g_{1} \mu^{2} & \left(f_{2}+\lambda\right) \mu^{3} & k_{3}-\mu^{4} & h_{4} \mu \\
h_{1} \mu & g_{2} \mu^{2} & \left(f_{3}+\lambda\right) \mu^{3} & k_{4}-\mu^{4} \\
k_{1}-\mu^{4} & h_{2} \mu & g_{3} \mu^{2} & \left(f_{4}+\lambda\right) \mu^{3}
\end{array}\right) .
$$

Taking determinant we obtain the polynomial

$$
p_{\lambda, \mu}=-\mu^{16}+K_{3}(\lambda) \mu^{12}+K_{2}(\lambda) \mu^{8}+K_{1}(\lambda) \mu^{4}+K_{0}(\lambda) .
$$

We present the explicit expressions for the polynomials $K_{i}(\lambda)$.

- $K_{3}(\lambda)=K_{33} \lambda^{3}+K_{32} \lambda^{2}+K_{31} \lambda+K_{30}$,

where

$$
\begin{aligned}
K_{33}= & f_{1}+f_{2}+f_{3}+f_{4}, \\
K_{32}= & g_{1}+g_{2}+g_{3}+g_{4}+f_{1} f_{2}+f_{1} f_{3}+f_{1} f_{4}+f_{2} f_{3}+f_{2} f_{4}+f_{3} f_{4}, \\
K_{31}= & h_{1}+h_{2}+h_{3}+h_{4}+f_{1} f_{2} f_{3}+f_{1} f_{2} f_{4}+f_{1} f_{3} f_{4}+f_{2} f_{3} f_{4}+f_{2} g_{4} \\
& +f_{3} g_{4}+f_{3} g_{1}+f_{4} g_{1}+f_{4} g_{2}+f_{2} g_{3}+f_{1} g_{2}+f_{1} g_{3}, \\
K_{30}= & k_{1}+k_{2}+k_{3}+k_{4}+f_{1} f_{2} f_{3} f_{4}+g_{1} g_{3}+g_{2} g_{4}+f_{2} h_{3}+f_{4} h_{1} \\
& +f_{1} h_{2}+f_{3} h_{4}+f_{2} f_{3} g_{4}+f_{3} f_{4} g_{1}+f_{1} f_{4} g_{2}+f_{1} f_{2} g_{3} .
\end{aligned}
$$

- $K_{2}(\lambda)=K_{22} \lambda^{2}+K_{21} \lambda+K_{20}$,

where

$$
\begin{aligned}
K_{22}= & -h_{2} h_{4}-g_{2} k_{3}-g_{3} k_{4}-h_{1} h_{3}-g_{1} k_{2}-k_{1} g_{4}, \\
K_{21}= & g_{2} h_{4} g_{3}-g_{1} k_{2} f_{4}-f_{1} h_{2} h_{4}-k_{1} g_{4} f_{3}-k_{1} f_{2} g_{4}-f_{1} g_{2} k_{3}-h_{2} h_{4} f_{3}-g_{1} k_{2} f_{3} \\
& +g_{1} g_{2} h_{3}+h_{1} g_{4} g_{3}+g_{1} h_{2} g_{4}-f_{1} g_{3} k_{4}-h_{1} f_{2} h_{3}-g_{2} k_{3} f_{4}-f_{2} g_{3} k_{4}-h_{2} k_{4} \\
& -h_{2} k_{3}-h_{1} h_{3} f_{4}-k_{2} h_{4}-h_{3} k_{4}-h_{1} k_{2}-h_{1} k_{3}-k_{1} h_{3}-k_{1} h_{4}, \\
K_{20}= & -k_{2} k_{4}-k_{2} k_{3}-k_{1} k_{2}-k_{1} k_{3}-k_{1} k_{4}-k_{3} k_{4}-k_{1} f_{2} g_{4} f_{3}-f_{1} h_{2} h_{4} f_{3} \\
& +f_{1} g_{2} h_{4} g_{3}-h_{1} k_{2} f_{4}-f_{1} g_{2} k_{3} f_{4}-f_{1} f_{2} g_{3} k_{4}-g_{1} g_{2} g_{4} g_{3}+g_{1} h_{2} g_{4} f_{3} \\
& -h_{1} f_{2} h_{3} f_{4}+g_{1} g_{2} h_{3} f_{4}+h_{1} f_{2} g_{4} g_{3}-g_{1} k_{2} f_{3} f_{4}-f_{1} h_{2} k_{3}-g_{1} k_{2} g_{3}-f_{1} h_{2} k_{4} \\
& -k_{1} g_{2} g_{4}+h_{1} h_{2} g_{4}-k_{2} h_{4} f_{3}-f_{2} h_{3} k_{4}+g_{2} h_{3} h_{4}-g_{2} g_{4} k_{3}-g_{1} g_{3} k_{4}+g_{1} h_{2} h_{3} \\
& -h_{1} k_{3} f_{4}+h_{1} h_{4} g_{3}-k_{1} f_{2} h_{3}-k_{1} h_{4} f_{3} .
\end{aligned}
$$

- $K_{1}(\lambda)=K_{11} \lambda+K_{10}$,

where

$$
\begin{aligned}
K_{11}= & h_{1} k_{2} k_{3}+h_{2} k_{3} k_{4}+k_{1} k_{2} h_{4}+k_{1} h_{3} k_{4}, \\
K_{10}= & h_{1} k_{2} k_{3} f_{4}+f_{1} h_{2} k_{3} k_{4}-k_{1} g_{2} h_{3} h_{4}+k_{1} g_{2} g_{4} k_{3}+k_{1} k_{2} h_{4} f_{3}+k_{1} f_{2} h_{3} k_{4} \\
& -h_{1} h_{2} g_{4} k_{3}+h_{1} h_{2} h_{3} h_{4}-h_{1} k_{2} h_{4} g_{3}-g_{1} h_{2} h_{3} k_{4}+g_{1} k_{2} g_{3} k_{4}+k_{1} k_{2} k_{4} \\
& +k_{1} k_{2} k_{3}+k_{2} k_{3} k_{4}+k_{1} k_{3} k_{4} .
\end{aligned}
$$

- $K_{0}(\lambda)=-k_{1} k_{2} k_{3} k_{4}$. 
Remark 2. We note that $K_{3}(\lambda)$ gives the polynomial invariants. Clearly $H_{1}=K_{33}=\operatorname{tr} L$. We also have $H_{2}=\frac{1}{2} \operatorname{tr} L^{2}=\frac{1}{2} K_{33}^{2}-K_{32}$ and $H_{3}=\frac{1}{3} \operatorname{tr} L^{3}=\frac{1}{3} K_{33}^{3}-K_{33} K_{32}+K_{31}$.

Finally, $H_{4}=\frac{1}{4} K_{33}^{4}+K_{33} K_{31}+\frac{1}{2} K_{32}-K_{30}-K_{33} K_{32}$.

These last relations hold provided that $k_{2}=k_{3}=k_{4}=0, h_{3}=h_{4}=0$ and $g_{4}=0$.

Remark 3. The next coefficient $K_{2}(\lambda)$ gives the rational invariants. We form the quotient $\frac{K_{21}}{K_{22}}$ and set $k_{2}=k_{3}=k_{4}=0$ and $h_{3}=h_{4}=0$. We obtain

$$
\frac{K_{21}}{K_{22}}=\frac{-g_{4}\left(k_{1} f_{3}+k_{1} f_{2}-h_{1} g_{3}-g_{1} h_{2}\right)}{-k_{1} g_{4}}=f_{2}+f_{3}-\frac{\left(h_{1} g_{3}+g_{1} h_{2}\right)}{k_{1}} .
$$

This is precisely $-i_{11}$.

Similarly, we form $\frac{K_{20}}{K_{22}}$ to obtain precisely $i_{21}$.

Remark 4. The last two terms, namely $K_{1}(\lambda)$ and $K_{0}(\lambda)$ become identically zero once we set $k_{2}=k_{3}=k_{4}=0, h_{3}=h_{4}=0$.

In the general case, the polynomial $p_{\lambda, \mu}$ involves polynomials $A(\lambda), B_{1}(\lambda), \ldots, B_{k}(\lambda)$ and $C_{1}(\lambda), \ldots, C_{s}(\lambda)$ with $k=\left[\frac{(n-1)}{2}\right]$ and $s=n-k-1$. The polynomial $A(\lambda)$ can be used to obtain the polynomial integrals. The polynomials $B_{i}(\lambda)$ give the rational integrals using the procedure described above and the $C_{j}(\lambda)$ vanish identically once we restrict to the phase space of the FKT lattice. A detailed proof of the general case will be given in a future publication.

[1] Bogoyavlensky O.I., On perturbations of the periodic Toda lattice, Comm. Math. Phys., 1976, V.51, $201-209$.

[2] Constantinides K., Generalized full Kostant-Toda lattices, Master Thesis, Department of Mathematics and Statistics, University of Cyprus, 2003.

[3] Damianou P.A., Paschalis P., Sophocleous C., A tri-Hamiltonian formulation of the full Kostant-Toda lattice, Lett. Math. Phys., 1995, V.34, 17-24.

[4] Damianou P.A., Multiple Hamiltonian structure of Bogoyavlensky-Toda lattices, Reviews Math. Phys., 2004, V.16, 175-241.

[5] Deift P.A., Li L.C., Nanda T., Tomei C., The Toda lattice on a generic orbit is integrable, Comm. Pure Appl. Math., 1986, V.39, 183-232.

[6] Ercolani N.M., Flaschka H., Singer S., The Geometry of the full Kostant-Toda lattice, in Colloque Verdier, Progress in Mathematics Series, Birkhaeuser Verlag, 1994, 181-225.

[7] Falqui G., Magri F., Pedroni M., Bi-Hamiltonian geometry, Darboux coverings, and linearization of the KP hierarchy, Comm. Math. Phys., 1998, V.197, 303-324.

[8] Faybusovich L., Gekhtman M., Elementary Toda orbits and integrable lattices, J. Math. Phys., 2000, V.41, 2905-2921.

[9] Flaschka H., On the Toda lattice, Phys. Rev., 1974, V.9, 1924-1925.

[10] Flaschka H., On the Toda lattice II. Inverse-scattering solution, Progr. Theor. Phys., 1974, V.51, 703-716.

[11] Flaschka H., Integrable systems and torus actions, Lecture Notes, University of Arizona, 1992.

[12] Fokas A.S., Fuchssteiner B., The Hierarchy of the Benjamin-Ono equations, Phys. Lett. A, 1981, V.86, $341-345$.

[13] Fuchssteiner B., Master symmetries and higher order time-dependent symmetries and conserved densities of nonlinear evolution equations, Progr. Theor. Phys., 1983, V.70, 1508-1522.

[14] Gelfand I.M., Zakharevich I., On the local geometry of a bi-Hamiltonian structure, in The Gelfand Mathematical Seminars 1990-1992, Editors L. Corvin et al., Boston, Birkhauser, 1993, 51-112.

[15] Henon M., Integrals of the Toda lattice, Phys. Rev. B, 1974, V.9, 1921-1923.

[16] Kostant B., The solution to a generalized Toda lattice and representation theory, Adv. Math., V.34, 195-338.

[17] Kupershmidt B., Discrete Lax equations and differential-difference calculus, Asterisque, 1985, V.123, 1-212.

[18] Magri F., A simple model of the integrable Hamiltonian equation, J. Math. Phys., 1978, V.19, $1156-1162$.

[19] Manakov S., Complete integrability and stochastization of discrete dynamical systems, Zh. Exp. Teor. Fiz., 1974, V.67, 543-555. 
[20] Meucci A., Compatible Lie algebroids and the periodic Toda lattice, J. Geom. Phys., 2000, V.35, $273-287$.

[21] Meucci A., Toda equations, bi-Hamiltonian systems, and compatible Lie algebroids, Math. Phys. Analysis Geom., 2001, V.4, 131-146.

[22] Moser J., Finitely many mass points on the line under the influence of an exponential potential - an integrable system, Lect. Notes Phys., 1976, V.38, 97-101.

[23] Moser J., Three integrable Hamiltonian systems connected with isospectral deformations, Adv. Math., 1975, V.16, 197-220.

[24] Singer S., The geometry of the full Toda lattice, Ph.D. Dissertation, Courant Institute, 1991.

[25] Toda M., One-dimensional dual transformation, J. Phys. Soc. Japan, 1967, V.22, 431-436. 